\title{
El tiempo social de las alianzas público privadas transnacionales en América Latina: un estudio de caso (2002-2010)
}

The social time of transnational public-private partnerships in Latin America: a case study (2002-2010)

\author{
Luz Rodríguez Escobar*
}

Resumen: A partir del caso del Proyecto de Experiencias en Innovación Social en América Latina y el Caribe, que diseñó y ejecutó la CEPAL y la Fundación W.K. Kellogg entre 2002 y 2010, esta investigación analiza el tiempo social de las alianzas público privadas para el desarrollo entre actores transnacionales que se establecieron en la región a inicios del siglo XXI. La categoría tiempo social se refiere a un constructo cultural que captura el ritmo social de los procesos y que permite identificar el rol jugado por cada actor dentro del sistema que ellos configuraron. Analizamos este caso desde la perspectiva de la historia del presente que tiene como acontecimiento inaugural la crisis de deuda externa de los ochenta.

Palabras clave: tiempo social, alianza público-privada, cooperación internacional, organizaciones transnacionales, América Latina.

\begin{abstract}
Based on the case of the Project of Experiences in Social Innovation in Latin America and the Caribbean, which was designed and executed by ECLAC and the W.K. Kellogg between 2002 and 2010, this research analyzes the social time of public-private partnerships for development between transnational actors that settled in the region at the beginning of the 21 st century. The social time category refers to a cultural construct that captures the social rhythm of the processes and that allows to identify the role played by each actor within the system that they configured. We analyzed tis case from the perspective of the history of the present that has as its inaugural event the external debt crisis of the eighties.
\end{abstract}

Keywords: social time, public-private partnership, international cooperation, transnational organizations, Latin America.

Recibido: 2 septiembre 2018 Aceptado: 8 noviembre 2018

\footnotetext{
${ }^{*}$ Colombiana. Doctoranda en historia. El artículo es parte de mi investigación doctoral en historia, que ha sido financiada con beca de la Universidad de Santiago de Chile. E-mail. luz.rodriguez.e@,usach.cl
} 


\section{Introducción}

Entre los años 2004 y 2010 se llevó a cabo el Proyecto de Experiencias en Innovación Social en América Latina y el Caribe (PEIS), que tuvo por objetivo identificar las prácticas locales o subnacionales innovadoras en términos sociales en la región. El mecanismo utilizado para tal fin fue la realización de un concurso de experiencias socialmente innovadoras, con el propósito de seleccionar las mejores y a partir de ellas generar recomendaciones de política pública para la región.

Los actores que tomaron parte en el PEIS fueron tres: la Comisión Económica para América Latina y el Caribe (CEPAL), que es un organismo internacional público; la Fundación W.K. Kellogg (FK), que es una entidad transnacional privada; y varias organizaciones socioeconómicas de base local presentes en los territorios de la región, que actuaron como participantes del concurso.

Este caso aconteció en el ciclo actual de la historia económica latinoamericana, donde los actores privados internacionales entran a desempeñar un rol destacado en la vida económica y social de la región y cuando mutaron las ideas respecto al desarrollo en la región, por lo que el PEIS adquiere un valor histórico para comprender la realidad social de América Latina en el tiempo presente.

El PEIS se ubica en una etapa de cooperación internacional marcada por los nuevos acuerdos globales que se gestaron al inicio del siglo XXI, en el que se reconoce al desarrollo social como prioritario en la agenda mundial. Ese consenso quedó expresado en los Objetivos de Desarrollo del Milenio (ODM), firmado por los países miembros de las Naciones Unidas en la Asamblea realizada en el año 2000, así como en la propuesta del Banco Mundial de lucha contra la pobreza en 2001.

El escalamiento político del tema social fue resultado de un amplio debate sobre los costos sociales de la crisis latinoamericana de la deuda externa que corrió a lo largo de los ochenta y noventa, donde organizaciones de las Naciones Unidas señalaron el negativo efecto social de políticas recomendadas por el Banco Mundial (BM) y organismos financieros internacionales. Dado que estos organismos condicionaron la ayuda financiera al cumplimiento de esas políticas, se justifica a enfatizar el carácter de imposición que asumieron esas medidas en la región.

El debate sobre los costos sociales de la crisis estuvo cruzado por otro, referente a las ideas de desarrollo, que desembocó en un enfrentamiento entre la CEPAL y el BM sobre estrategia de desarrollo para la región. En la década de los ochenta, los planteamientos estructuralistas de la CEPAL apuntaban a medidas de impulso a la industrialización guiada por el Estado, mientras que el BM impulso otra centrada en la promoción de las exportaciones y orientada por el mercado. A partir de 1990, la CEPAL modificó sus planteamientos y su propuesta de desarrollo. Este cambio fue nominado por la propia CEPAL como el paso del estructuralismo al neoestructuralismo, que dio como resultado un acercamiento del pensamiento de la CEPAL con las posiciones del BM, especialmente en materia del mayor protagonismo del mercado y el cambio en el papel del Estado. Sin embargo, en materia de desarrollo social se evidenciaron diferencias.

En ese contexto surge, en 2002, el convenio entre la FK y la CEPAL para diseñar y ejecutar el PEIS, que es el objeto de análisis de esta investigación. Su análisis se aborda desde la corriente historiográfica de la historia del presente, la cual se propone dar cuenta de la experiencia vivida por la generación que la experimentó, otorgando importancia a la acción de los actores, pero dentro de una relación dialéctica con la estructura en la que se produce. Esta perspectiva permite remitirse a la memoria, con su componente subjetivo, que busca objetivarse al ubicarla en las trayectorias históricas que captan los procesos en que está inmersa ${ }^{1}$.

${ }^{1}$ Julio Aróstegui, La historia vivida, sobre la historia del presente, Madrid, Alianza, 2004. 
El artículo tiene por objetivo comprender la manera que se estructuran las relaciones entre actores transnacionales y otros actores que se sientan en América latina, alrededor de iniciativas de cooperación internacional para el desarrollo, desde un enfoque histórico en el tiempo presente.

La investigación parte de la hipótesis que el PEIS fue un caso atípico dentro de la CEPAL, que opero como una especie de proyecto enclave de una transnacional privada dentro de un organismo público. La categoría elegida para captar esas relaciones es el tiempo social ${ }^{2}$ que es un constructo cultural para capturar los ritmos sociales de los procesos impulsados por grupos humanos y también permite identificar el rol de cada sujeto dentro del sistema que ellos configuran.

Por tanto, la metodología de investigación considera al PEIS como un sistema que tuvo una estructura y un ritmo y parte por establecer los momentos emblemáticos o hitos del proyecto, para luego describirlos y analizar cada uno, con el foco en el actor que más influyó en su configuración y la manera en que ejerció ese dominio.

El documento se estructura en cinco secciones. La primera es la presente introducción; la segunda detalla el contexto económico, político y social de América Latina que caracteriza el actual ciclo de historia económica; la tercera detalla el origen del caso de estudio y los actores que lo protagonizan; la cuarta analiza el tiempo social del PEIS a partir de sus hitos. La quinta presenta las conclusiones.

\section{América Latina al inicio del siglo XXI}

Los países de América Latina experimentaron fuertes transformaciones sociales, económicas y políticas durante las dos últimas décadas del siglo XX. En el plano económico se destacó el cambio de estrategia de desarrollo y la inserción de las economías nacionales al mercado internacional. Para Bértola y Ocampo ${ }^{3}$ esos cambios marcan en tránsito de un ciclo de historia económica marcado por la industrialización impulsada por el Estado a otro, caracterizado por la promoción de las exportaciones y orientación hacia el mercado, que impulsó la entrada de la región a la reciente era de globalización.

El nuevo ciclo histórico también puede ser considerado la génesis de la historia del presente de América Latina, marcada por la crisis de deuda externa que tiene como evento inicial la declaración de moratoria de México en 1982. Alrededor de problema de la deuda externa se conjugaron diversos factores, tanto internos a la región como externos, que hicieron de ella el acontecimiento que inaugura el tiempo presente de América Latina.

Entre los factores externos, cabe resaltar la presión que ejercieron los bancos privados internacionales, con el apoyo de los gobiernos de los países centrales y de los organismos financieros internacionales, para obligar a que los países latinoamericanos negociar individualmente su deuda e impedir que configuraran un cartel de deudores ${ }^{4}$. Sin embargo, los acreedores sí pudieron configurar un cartel $^{5}$, que tuvo como coordinadores al FMI y el BM. El Banco Interamericano de Desarrollo (BID) actuaba dentro de la región en la misma línea del BM.

2 Aron Gurevitch, "El tiempo como un problema de historia cultural”, Paul Ricoeur, Claude Larre, Raimundo Panikkar, Alexis Kagame, G.E.R Lloyd, André Neher, Germano Pattaro, Louis Gardet, y Aron Guervitch (eds), Las culturas y el tiempo, Salamanca, UNESCO, 1979, 260-281.

3 Luís Bértola y José Antonio Ocampo, El desarrollo de América latina desde la independencia, México, Fondo de Cultura Económica, 2013.

4 José Antonio Ocampo, "La crisis latinoamericana de la deuda a la luz de la historia", José Antonio Ocampo, Bárbara Stalling, Ines Bustillo, Helvia Belloso y Robert Frenkel (eds), La crisis latinoamericana de la deuda desde una perspectiva histórica, Santiago, Naciones Unidas, 2014, 19-49.

5 Bárbara Stalling, "La economía política de las negociaciones de la deuda: América Latina en la década de los ochenta", José Antonio Ocampo, Bárbara Stalling, Inés Bustillo, Helvia Belloso y Robert Frenkel (eds), La crisis latinoamericana de la deuda desde una perspectiva bistórica, Santiago, Naciones Unidas, 2014, 53-82. 
A inicios de la década de los ochenta, los organismos financieros internacionales aprovecharon la necesidad de financiamiento externo de los países latinoamericanos para instalar políticas de estabilización y de ajuste estructural, como condición a la entrega de la ayuda financiera. Estas "recomendaciones" de política tenían una clara orientación a favor del libre mercado y la reducción del papel del Estado, que se sustentaban en la creencia en el mercado como eje articulador de todos los procesos económicos. Para efectos del tema que aquí se aborda cabe destacar aquellas políticas dirigidas a reducir los déficits fiscales por la vía del recorte del gasto público, así como la privatización de las empresas públicas y las medidas a favor de la inversión extranjera, que favorecieron la mayor presencia de entidades transnacionales en la región.

En 1990 Williamson ${ }^{6}$ denominó a esas las políticas de estabilización y ajuste estructural el Consenso de Washington, concepto que resalta la diversidad de actores, con sede en esa ciudad, que respaldaban esas medidas. En ellas, sobresalen el gobierno de Estados Unidos y los organismos financieros internacionales, pero también fueron actores claves los banqueros privados internacionales, las organizaciones de empresarios transnacionales y los centros de investigación (think tank en inglés), que contribuyeron a instalar la fe en el mercado como solución a los males económicos de la época.

A medida que instalaron en la región las nuevas políticas, se materializaron reformas estructurales que afectaron la vida económica y social de la región, entre ellos el aumento de la pobreza y la desigualdad social. Algunos de esos costos sociales fueron denunciados por organizaciones internacionales de las Naciones Unidas, como la UNICEF, el PNUD y la CEPAL.

A fines de la década de los ochenta ocurrió el fin de la guerra fría, con la transición de los países socialistas de Europa oriental al capitalismo y la transición de los gobiernos militares de la región a regímenes democráticos. En este nuevo contexto internacional, las denuncias sobre los costos negativos del CW condujo a que la cooperación internacional de los países centrales a los países de la región asumiera la figura de subvenciones a organizaciones no gubernamentales de desarrollo (ONGD) para que se hicieran cargo programas sanitarios y educativos que los estados habían dejado de atender debido al recorte de gasto público y el desmantelamiento del Estado.

El debate sobre los costos sociales de la crisis estuvo antecedido por otro, referente a las ideas de desarrollo, que desembocó en un enfrentamiento entre la CEPAL y el FMI y BM, sobre estrategias y políticas de desarrollo para la región. Los planteamientos estructuralistas de la CEPAL sobre industrialización guiada por el Estado fueron desplazados por políticas del CW y dentro de la misma CEPAL se desató una disputa, pues los problemas de la crisis de deuda externa pusieron de manifiesto que la propuesta de la CEPAL carecía de elementos para enfrentar los problemas monetarios y financieros de costo plazo.

A partir de 1990 la CEPAL replanteó su enfoque y cambió su pensamiento del estructuralismo al neoestructuralismo ${ }^{7}$. Este giro fue interpretado por las nuevas autoridades de la CEPAL como una renovación de su pensamiento para adaptarse a las nuevas circunstancias, que se plasmó en una nueva estrategia de desarrollo para la región, nombrada como transformación productiva con equidad. La nueva estrategia de la CEPAL se acercó a los postulados del BM en el énfasis dado a la promoción las exportaciones, pero le agregó el componente de la equidad, como factor que contribuye a la competitividad. Además, el neoestructuralismo reposicionó el rol del Estado, ya no como líder del proceso económico sino regulador del proceso social y político que acompaña al proceso económico. De esa manera CEPAL se coloca en una posición crítica pero no opuesta a los planteamientos del BM y FMI.

6 John Williamson, "Lo que Washington quiere decir cuando se refiere a reformas de las políticas económicas", Manuel Guitián y Joaquim Muns (eds), La cultura de la estabilidad y el consenso de Washington, Barcelona, La Caixa, 1999, 67-118.

7 Ricardo Bielschowsky, "Sesenta años de la CEPAL: estructuralismo y neoestructuralismo", Revista de la CEPAL, 1998, 173194. 
En 1995 tuvo lugar la primera cumbre de desarrollo social, en el que se resaltó la urgencia y gravedad del deterioro social. Se resaltó el aumento de la pobreza y de la desigualdad en el mundo y por supuesto en América Latina. Según cifras de la CEPAL', en 1990 el 48,4\% de la población latinoamericana vivía en condiciones de pobreza, un fenómeno aún más alarmante en las zonas rurales donde la pobreza llegó al 65,2\%. Durante la década de los ochenta el número de pobres aumentó, al pasar de 136 millones en 1980 a 204 millones en 1990. En materia de empleo el panorama también fue alarmante ya que el desempleo creció considerablemente, al pasar del 6,6\% en 1980 al 11\% en $19999^{9}$. En términos de distribución del ingreso, en las décadas de los ochenta y noventa la tendencia fue a la concentración de la riqueza.

Ante esa realidad social reaccionaron los gobiernos del mundo. En 2000 la Asamblea de las Naciones Unidas generó un acuerdo mundial, que ubicó a la pobreza como el principal desafío social del planeta. Ese consenso quedó expresado en los Objetivos de Desarrollo del Milenio (ODM) y en la propuesta del BM de lucha contra la pobreza, que fue el tema del Informe de Desarrollo Mundial de 2001. La pobreza, se convertía en una prioridad de la agenda internacional. Así mismo, los ODM estuvieron acompañados por el reconocimiento del BM sobre su responsabilidad en el aumento de la pobreza y el de que las políticas del CW no garantizaban el desarrollo.

"Si bien los desafíos actuales y futuros continúan siendo sobrecogedores, entramos el nuevo milenio con una comprensión más adecuada del desarrollo. Hemos aprendido que los elementos tradicionales de las estrategias para fomentar el crecimiento -estabilidad macroeconómica y reformas favorables al mercado- son fundamentales para reducir la pobreza. Pero ahora comprendemos también la necesidad de hacer mayor hincapié en sentar las bases institucionales y sociales para el proceso de desarrollo y en corregir la vulnerabilidad y alentar la participación para llegar a un crecimiento integrador" 10 .

Además, los ODM se complementaron con un pacto para el desarrollo que ampliaba los espacios de participación del sector privado e invitaba a los empresarios a asumir un rol más activo, que tomó la forma de Responsabilidad Social Empresarial y alianzas público privadas para el desarrollo. Es en ésta época que las alianzas público-privadas empezaron a convertirse en protagonistas del desarrollo.

Para el sector privado, la lucha contra la pobreza fue considerada una oportunidad de negocio para desarrollar mercados de bajo costo con potencial alto de retornos ${ }^{11}$. En otras palabras, una oportunidad de crear bienes y servicios baratos para pobres, bajo la perspectiva de la gerencia de negocios (The management).

En ese sentido, a inicios del siglo también ocurrió un cambio conceptual que refleja un cambio en la manera en que entidades filantrópicas conciben su actividad. Ya sus aportes a la sociedad no se consideraron meras donaciones sino que se concebían como una inversión social ${ }^{12}$. El cambio es importante porque logra vincular la provisión de recursos privados para fines sociales con propósitos de rentabilidad, es decir en vez de donaciones lo que se proponen hacer es inversiones que ofrezcan retornos sociales junto con retornos financieros.

De manera que las instituciones sin ánimo de lucro entran en la lógica de ocuparse en lograr la sustentabilidad financiera de sus instituciones y de los proyectos que patrocinan. Esos aportes de la comunidad internacional a la cooperación internacional al desarrollo, tuvieron la característica de atarse al CW puesto que la entrega de aportes obligaba a que los países receptores cumplieran con las medidas

8 CEPAL, Panorama social, Santiago, Naciones Unidas, 2010.

${ }^{9}$ CEPAL, Panorama social, Santiago, Naciones Unidas, 2009.

${ }^{10}$ Banco Mundial, Informe sobre el desarrollo mundial 2000/200: lucha contra la pobreza, Madrid, Mundi-Prensa, 2001, vi.

${ }_{11}$ C.K. Prahalad y Stuart Hard, "The Fortune at the bottom of the pyramid”, Security and strategy, 2002.

12 Maximillian Martin, "Cuatro revoluciones en la filantropía global”, Working papers, Ginebra, Impact Economy, 2011. 
del CW. Al respecto, Alejandra Boni13 señala que la cooperación para el desarrollo otorgó un nuevo impulso al CW y contribuyó a erguirlo como única alternativa para organizar la vida económica, a la vez que contribuyó a consolidar la idea del intervencionismo estatal como obstáculo al buen funcionamiento de la economía. De modo que la cooperación internacional contribuyó a favorecer el orden mundial que configuro el CW.

Pero en América Latina ese orden mundial empezó a ser cuestionado y, hasta cierto punto, desafiado en los últimos años de la década de los noventa y especialmente al inicio del siglo XXI. El nuevo milenio inicia con la expresión en las urnas del descontento social y la oposición social a las políticas neoliberales. Para sorpresa del mundo, que recién en 1989 había presenciado la caída del bloque socialista y la transición de esas economías al capitalismo, en América Latina la sociedad elegía como gobernantes a dirigentes con discursos críticos al capitalismo y de corte socialista. Fue el caso de Hugo Chaves (1999) en Venezuela, Néstor Kirchner (2003) en Argentina, Ignacio Lula Da Silva (2003) en Brasil, Tabaré Vásquez (2005) en Uruguay, Evo Morales (2006) en Bolivia, Michelle Bachelet (2006) en Chile y Rafael Correa (2007) en Ecuador.

Para Fisher y Plehwe ${ }^{14}$ ese giro a la izquierda de algunos países latinoamericanos se puede interpretar como una reacción en contra de los gobiernos de derecha que habían dominado en las décadas anteriores y del modelo económico liberal o neoliberal. También puede interpretarse como una reacción en contra de las múltiples promesas rotas de desarrollo y los altos niveles de pobreza y desigualdad que exhibían la región en las dos últimas décadas del siglo XX. En todo caso, a inicios del siglo XXI América Latina abrió nuevos horizontes de expectativa en la región, con posibilidades de futuro no capitalista o por lo menos con variedades de capitalismos. En la región había sociedades dispuestas a ensayar nuevos rumbos.

De manera que América Latina terminó el siglo XX con una tendencia al empobrecimiento de la población, con un manejo económico enfocado a superar la crisis de deuda externa, con una cooperación internacional que le impuso condiciones y con transiciones democráticas, que permitió a las sociedades expresar su malestar en las urnas. En ese escenario, nos proponemos analizar las relaciones entre los actores que interactúan en el PEIS.

\section{El PEIS y sus actores}

El PEIS fue un proyecto que se llevó a cabo en la CEPAL entre los años 2002 y 2010. Tuvo origen en una iniciativa de la FK en 2002 cuando esta entidad realizaba los preparativos para celebrar su aniversario 75 y estableció que su forma de celebrar sería por medio de donaciones significativas de carácter regional.

Para el caso de América Latina, la FK eligió como socio estratégico a la CEPAL, con quien firmó un primer acuerdo de un año para formular un proyecto que tuviera como actividad central la realización de un concurso de experiencias en innovación social. Luego, en 2004, firmó un segundo convenio para ejecutar el concurso por cuatro años. Finalmente, en 2008 extiende el convenio por dos años más, con cierre definitivo en 2010.

En el primer convenio, ambas entidades acordaron los términos del concurso y decidieron convocar a participar a "los gobiernos locales o municipales, las organizaciones no gubernamentales, las

\footnotetext{
13 Alejandra Boni, "El sistema de la cooperación internacional al desarrollo. Evolución histórica y retos actuales", Carola Calabuig Torno y María de los Llanos Gómez-Torres (coords), La cooperación internacional para el desarrollo, Cuadernos de cooperación para el desarrollo No 1, Valencia, Univeristat politécnica de Valencia, 2010, 7-52.

${ }^{14}$ Karin Fischer y Dieter Plehwe, "Redes de think tank e intelectuales de derecha en América Latina", Nueva sociedad, 215, 2013, $70-86$.
} 
organizaciones de las propias comunidades, y las comunidades religiosas en cualquiera de los 33 países de América Latina y el Caribe miembros de la CEPAL"15.

Las áreas temáticas a las que se convocó a concursar fueron: generación de ingresos, desarrollo rural y agrícola, salud comunitaria, educación básica, seguridad alimentaria y nutrición, programa de juventud, responsabilidad social y voluntariado. Estas temáticas fueron reagrupadas al final del concurso en 4: generación de ingresos, juventud en riesgo, afirmación de derechos y voluntariado y participación comunitaria.

El concurso realizó 5 ciclos anuales durante los cuales se postularon 4800 experiencias, de las cuales fueron admitidas 3000. En cada ciclo un jurado internacional seleccionó y premió a las cinco mejores ${ }^{16}$, las cuales recibieron un premio en dinero: el primer lugar recibió 10.000 dólares y las siguientes 8.000, 5.000, 3.000 y 2.000 dólares respectivamente.

Entre la CEPAL y la FK acordaron los criterios de postulación y el mecanismo de selección de las experiencias ganadoras. Los concursantes debían postular a través del envío de un formulario en donde registraban su experiencia, esos formularios eran recepcionados por el equipo del proyecto que se configuró dentro de la CEPAL, luego pasaban a evaluadores externos expertos en cada una de las temáticas. A las experiencias mejor evaluadas se les visitaba en terreno y de ahí se elegían las 25 experiencias finalistas de cada ciclo, las que eran invitadas a presentar sus trabajos ante un jurado de expertos, que seleccionaba las cinco ganadoras.

Desde el inicio del concurso, los criterios para seleccionar las experiencias ganadoras fueron: la incorporación de cambios sensibles que modifican y enriquecen prácticas tradicionales de generación de recursos y capacidades; el impacto en la calidad de vida de los beneficiarios; la creación, el desarrollo y la consolidación de procesos de participación de la comunidad, es decir, el fortalecimiento de la ciudadanía y, a través de ella, de la democracia; la replicabilidad de las experiencias y su sostenibilidad. A continuación, se caracterizan los tres tipos de actores que participaron del PEIS.

\subsection{E1 actor transnacional privado: 1a FK}

La FK es una entidad transnacional privada con sede principal en Michigan, Estados Unidos, dedicada a la filantropía. Fue creada en 1930 por Will Keith Kellogg, el mismo pionero de los cereales Kellogg, una compañía multinacional agroalimentaria que para inicios del silgo XXI figuraba entre las diez empresas que controlan el consumo masivo de bienes en el mundo. La FK ha tenido presencia en América Latina desde 1941 con programas de becas para que profesionales latinoamericanos del área médica se especializaran en Estados Unidos ${ }^{17}$. En 2002, la FK proyectó celebrar su aniversario 75 por medio de donaciones emblemáticas y para el caso de América Latina.

En ese marco, el Director de la FK para América Latina, Francisco Tancredi, ideó la realización de un concurso para identificar experiencias que fueran exitosas en innovación social, que tuviera cobertura regional. Para llevar a cabo esa iniciativa buscó un socio dentro de la región y eligió a la CEPAL como socio estratégico.

\footnotetext{
15 CEPAL, Palabras del Sr, José Luis Machinea, Secretario ejecutivo de la CEPAL, en ceremonia de lanzamiento del Concurso de innovación social [online], Concurso de innovación social, Santiago, 2004 [consultado en diciembre de 2014], Disponible en internet: <URL:http://www.eclac.cl/dds/innovacionsocial/e/concurso.ttm>.

16 Adolfo Rodríguez y Hernán Alvarado, Claves de innovación social en América Latina y el Caribe, Santiago, Naciones Unidas, 2008.

${ }^{17}$ Fundación W.K.Kellogg, Annual Report: A legacy of innovation, Michigan, W.K. Kellogg, 2004.
} 


\subsection{El actor internacional público: la CEPAL}

La CEPAL es un organismo internacional público, dependiente de la Organización de las Naciones Unidas, responsable de promover el desarrollo económico y social de América Latina y el Caribe, que fue fundado en 1948. Funciona como un centro de estudio en la región que colabora con los Estados miembros y diversas instituciones locales, nacionales e internacionales en el análisis de los procesos de desarrollo a través de la formulación, seguimiento y evaluación de las políticas públicas. Cabe destacar que entre sus Estados miembros están 33 países latinoamericanos y 5 países de fuera de la región (Estados Unidos, Canadá, Francia, reino Unido y Países Bajos). Tiene su sede central en Santiago, Chile.

En 2016 la CEPAL fue señalada como uno de los principales tanques de pensamiento de América Latina ${ }^{18}$. Sin embargo, a inicios del 2000 su rol como orientador de políticas públicas estaba desdibujado porque en la década anterior había cambiado su línea de pensamiento, al pasar de un enfoque estructuralista a otros neoestructuralista, que aún estaba en construcción. En todo caso, como miembro de Naciones Unidas, estaba obligado a mostrar resultados en términos de las metas fijadas por los ODM.

\subsection{Los actores locales}

A lo largo de los cinco ciclos del PEIS fueron examinadas 3000 experiencias, de las cuales fueron premiadas 25 por ser las más innovadoras socialmente. La Tabla 1 registra las experiencias premiadas.

Tabla 1 Proyectos ganadores del Concurso Experiencias en Innovación Social

\begin{tabular}{|c|c|c|c|c|}
\hline $\begin{array}{l}\text { País y fecha } \\
\text { de origen }\end{array}$ & Territorio & Experiencia & Organización & Concurso \\
\hline Haití (1999) & $\begin{array}{l}\text { Limonade, Dpto de } \\
\text { Norte }\end{array}$ & Let Agogo /leche en abundancia & VETERIMED & Primer lugar 2005 \\
\hline $\begin{array}{l}\text { Argentina } \\
(1996)\end{array}$ & $\begin{array}{l}\text { Quebrada de } \\
\text { Humahuaca/ } \\
\text { Provincias de Jujuy y } \\
\text { de Salta }\end{array}$ & $\begin{array}{l}\text { Programa integrado de cultivos } \\
\text { Andinos }\end{array}$ & $\begin{array}{l}\text { Cooperativa } \\
\text { Agropecuaria } \\
\text { Artesanal Unión } \\
\text { Quebrada y Valle / } \\
\text { CAUQUEVA }\end{array}$ & Segundo lugar 2005 \\
\hline $\begin{array}{l}\text { Guatemala } \\
\text { (1995) }\end{array}$ & $\begin{array}{l}\text { Ciudad de Guatemala } \\
\text { y municipios aledaños }\end{array}$ & $\begin{array}{l}\text { Prevención del fenómeno de } \\
\text { droga y mara en áreas urbano } \\
\text { marginales y rurales }\end{array}$ & $\begin{array}{l}\text { Asociación Grupo } \\
\text { Ceiba }\end{array}$ & Tercer lugar 2005 \\
\hline Brasil (1986) & $\begin{array}{l}\text { Voria de Santo } \\
\text { Antao, Estado de } \\
\text { Pernambuco }\end{array}$ & Ecoorganica & $\begin{array}{l}\text { Ecoorgania - } \\
\text { cooperativa de } \\
\text { agricultores }\end{array}$ & Cuarto lugar 2005 \\
\hline Brasil (2005) & $\begin{array}{l}\text { Municipios de } \\
\text { Belterra, Aveiro y } \\
\text { Santarém, Estado de } \\
\text { Pará }\end{array}$ & $\begin{array}{l}\text { Acciones de salud comunitaria en } \\
\text { la Floresta Nacional de Tapajós }\end{array}$ & $\begin{array}{l}\text { Centro de Estudios } \\
\text { Avanzados de } \\
\text { Promoción Social y } \\
\text { Ambiental (CEAPS), } \\
\text { Proyecto Salud y } \\
\text { Alegría (PSA) }\end{array}$ & Quinto lugar 2005 \\
\hline Perú (2000) & $\begin{array}{l}\text { Departamento de } \\
\text { Cusco }\end{array}$ & $\begin{array}{l}\text { Defensorías comunitarias: una } \\
\text { respuesta comunitaria a la }\end{array}$ & $\begin{array}{l}\text { Instituto de Defensa } \\
\text { Legal }\end{array}$ & Primer lugar 2006 \\
\hline
\end{tabular}

${ }^{18}$ Joseph McGann, Global go to think tank index report, Pennsylvania University, 2016. 


\begin{tabular}{|c|c|c|c|c|}
\hline & & violencia familiar & & \\
\hline $\begin{array}{l}\text { Argentina } \\
(1996)\end{array}$ & $\begin{array}{l}\text { La plata, Provincia de } \\
\text { Buenos Aires }\end{array}$ & $\begin{array}{l}\text { Sistema de sostén para } \\
\text { adolescentes tutelados }\end{array}$ & $\begin{array}{l}\text { Procuraduria General } \\
\text { de Poder judicial- } \\
\text { Provincia de Buenos } \\
\text { Aires }\end{array}$ & Segundo lugar 2006 \\
\hline Perú (1986) & $\begin{array}{l}\text { Distrito Ocuviri, } \\
\text { Lampa, Puno }\end{array}$ & $\begin{array}{l}\text { Producción sostenible de truchas } \\
\text { en el sistema extensivo e intensivo } \\
\text { de lagunas y jaulas }\end{array}$ & $\begin{array}{l}\text { Asociación pesquera } \\
\text { "Flor de Llankakahua" }\end{array}$ & Tercer lugar 2006 \\
\hline $\begin{array}{l}\text { Paraguay } \\
(2000)\end{array}$ & $\begin{array}{l}\text { Fram, Departamento } \\
\text { de Itapúa }\end{array}$ & $\begin{array}{l}\text { Programa comunitario de salud- } \\
\text { Salud responsabilidad de todos }\end{array}$ & $\begin{array}{l}\text { Consejo Legal de Salud } \\
\text { de Fram }\end{array}$ & Cuarto lugar 2006 \\
\hline $\begin{array}{r}\text { Brasil } \\
(1995)\end{array}$ & $\begin{array}{l}\text { Senhor do Bonfim, } \\
\text { Estado de Bahía }\end{array}$ & Travesuras infantiles & $\begin{array}{l}\text { Grupo Joven de } \\
\text { Asistencia Social del } \\
\text { Senhor do Bonfim }\end{array}$ & Quinto lugar 2006 \\
\hline $\begin{array}{r}\text { Brasil } \\
(1998)\end{array}$ & $\begin{array}{l}\text { Sobral, Estado de } \\
\text { Ceará }\end{array}$ & $\begin{array}{l}\text { Trébol de cuatro hojas: estrategia } \\
\text { de reducción de la morbilidad } \\
\text { materna, perinatal e infantil }\end{array}$ & $\begin{array}{l}\text { Secretaria de Salud y } \\
\text { Acción Social de } \\
\text { Sobral }\end{array}$ & Primer lugar 2007 \\
\hline $\begin{array}{l}\text { Bolivia } \\
(2006)\end{array}$ & $\begin{array}{l}\text { Departamentos de La } \\
\text { Paz y Potosí }\end{array}$ & Hospedaje estudiantil en familia & $\begin{array}{l}\text { Fundación Pueblo y } \\
\text { municipios }\end{array}$ & Segundo lugar 2007 \\
\hline $\begin{array}{l}\text { Ecuador } \\
(2002)\end{array}$ & $\begin{array}{l}\text { Parroquia de San } \\
\text { Rafael de la Laguna }\end{array}$ & $\begin{array}{l}\text { Manejo y tratamiento de aguas } \\
\text { residuales con lenteja acuática, } \\
\text { lechugín y totora }\end{array}$ & $\begin{array}{l}\text { Centro de Estudios } \\
\text { Pluriculturales, } \\
\text { CEPCU }\end{array}$ & Tercer lugar 2007 \\
\hline Brasil (1989) & $\begin{array}{l}\text { Nova California, } \\
\text { Estado de Rondonia }\end{array}$ & $\begin{array}{l}\text { Reforestación Económica Unida y } \\
\text { Adensada }\end{array}$ & $\begin{array}{l}\text { Asociación de los } \\
\text { pequeños agro } \\
\text { silvicultores, RECA }\end{array}$ & Cuarto lugar 2007 \\
\hline Brasil (2003) & $\begin{array}{l}\text { Belo Horizonte, } \\
\text { Minas Gerais }\end{array}$ & $\begin{array}{l}\text { Programa de erradicación del } \\
\text { trabajo infantil y protección de los } \\
\text { adolescentes en el trabajo } \\
\text { doméstico }\end{array}$ & Circo de todo Mundo & $\begin{array}{l}\text { Quinto lugar } 2007 \\
\text { Empate }\end{array}$ \\
\hline $\begin{array}{l}\text { Argentina } \\
(1997)\end{array}$ & $\begin{array}{l}\text { San Carlos de } \\
\text { Bariloche }\end{array}$ & $\begin{array}{l}\text { Programa de prevención de } \\
\text { violencia a través de proyectos de } \\
\text { resolución de conflictos mediante } \\
\text { pares en escuelas con niños y } \\
\text { jóvenes en riesgo y en grupos de } \\
\text { educación no formal }\end{array}$ & $\begin{array}{l}\text { Fundación Alternativa } \\
\text { Social y Educativa }\end{array}$ & $\begin{array}{l}\text { Quinto lugar } 2007 \\
\text { Empate }\end{array}$ \\
\hline $\begin{array}{l}\text { Belice } \\
(2005)\end{array}$ & $\begin{array}{l}\text { Punta Gorda, Capital } \\
\text { del Distrito de Toledo }\end{array}$ & $\begin{array}{l}\text { Copa de agua fresca de la Liga } \\
\text { Medioambiental de Futbol }\end{array}$ & $\begin{array}{l}\text { Toledo Institute for } \\
\text { Development and } \\
\text { Environment (TIDE) }\end{array}$ & Primer lugar 2008 \\
\hline $\begin{array}{l}\text { Colombia } \\
(2001)\end{array}$ & $\begin{array}{l}\text { Bogotá y municipios } \\
\text { de Cundinamarca }\end{array}$ & $\begin{array}{l}\text { Programa para la gestión del } \\
\text { conflicto escolar, Hermes }\end{array}$ & $\begin{array}{l}\text { Cámara de Comercio } \\
\text { de Bogotá }\end{array}$ & Segundo lugar 2008 \\
\hline $\begin{array}{l}\text { Ecuador } \\
(2005)\end{array}$ & Azuay y Cañar & $\begin{array}{l}\text { Fortalecimiento de las finanzas } \\
\text { populares en Azuay y Cañar }\end{array}$ & $\begin{array}{l}\text { Fondo Ecuatoriano } \\
\text { Populorum progressio }\end{array}$ & Tercer lugar 2008 \\
\hline
\end{tabular}




\begin{tabular}{|c|c|c|c|c|}
\hline Chile (2007) & Región metropolitana & $\begin{array}{l}\text { Del campamento al barrio. } \\
\text { Implementación de mesas } \\
\text { participativas de trabajo en los } \\
\text { campamentos de la Región } \\
\text { Metropolitana por parte de los } \\
\text { pobladores y voluntario de un } \\
\text { techo para Chile. }\end{array}$ & $\begin{array}{l}\text { Un techo para Chile } \\
\text { (UTPCH) }\end{array}$ & Cuarto lugar 2008 \\
\hline $\begin{array}{l}\text { Argentina } \\
(2003)\end{array}$ & $\begin{array}{l}\text { Tilcara, Provincia de } \\
\text { Jujuy }\end{array}$ & $\begin{array}{l}\text { Nuestras huellas. Una experiencia } \\
\text { de construcción del aprendizaje } \\
\text { desde la escuela tradicional hacia } \\
\text { la interculturalidad }\end{array}$ & $\begin{array}{l}\text { Escuela Normal Dr. } \\
\text { Eduardo Casanova }\end{array}$ & Quinto lugar 2008 \\
\hline $\begin{array}{r}\text { Brasil } \\
(2004) \\
\end{array}$ & $\begin{array}{l}\text { Maringá, Estado de } \\
\text { Paraná }\end{array}$ & Observatorio social de Maringá & $\begin{array}{l}\text { Sociedad Éticamente } \\
\text { Responsable - SER }\end{array}$ & Primer lugar 2009 \\
\hline $\begin{array}{l}\text { México } \\
(2001)\end{array}$ & $\begin{array}{l}\text { Ayoquezco de } \\
\text { Aldama, Oaxaca }\end{array}$ & $\begin{array}{l}\text { Proyecto binacional de inversión } \\
\text { de remesas para el establecimiento } \\
\text { de una planta procesadora de } \\
\text { alimentos nostálgicos de Oaxaca } \\
\text { en Ayoquezco del Aldama }\end{array}$ & $\begin{array}{l}\text { Fundación para la } \\
\text { productividad del } \\
\text { campo, A.C. } \\
\text { FUPROCA }\end{array}$ & Segundo lugar 2009 \\
\hline $\begin{array}{l}\text { Costa Rica } \\
(2003)\end{array}$ & Cantón de Coto Brus & $\begin{array}{l}\text { Atención en salud integral a la } \\
\text { población indígena altamente } \\
\text { móvil }\end{array}$ & $\begin{array}{l}\text { Caja Costarricense de } \\
\text { Seguridad Social }\end{array}$ & Tercer lugar 2009 \\
\hline Chile (1998) & $\begin{array}{l}\text { Provincia del Elqui, } \\
\text { Coquimbo }\end{array}$ & $\begin{array}{l}\text { De la basura a la rehabilitación, } \\
\text { una esperanza integradora }\end{array}$ & $\begin{array}{l}\text { Unió de padres y } \\
\text { amigos solidarios } \\
\text { (UPASOL) }\end{array}$ & Cuarto lugar 2009 \\
\hline $\begin{array}{l}\text { Argentina } \\
(1999)\end{array}$ & $\begin{array}{l}\text { Resistencia, Provincia } \\
\text { del Chaco }\end{array}$ & Abuelas cuentacuentos & $\begin{array}{l}\text { Fundación Mempo } \\
\text { Giardinelli }\end{array}$ & Quinto lugar 2009 \\
\hline
\end{tabular}

Fuente: Elaboración propia, con base en CEPAL, Banco de experiencias en innovación social, 2010.

Las iniciativas ganadoras provinieron de territorios localizados en 13 países de la región, con presencia mayoritaria de Brasil y Argentina. Se destaca que la mitad de las experiencias ganadoras fueron presentadas por organizaciones no gubernamentales $(\mathrm{ONG})$ que desarrollaban proyectos con la participación de las comunidades y que en algunos casos estaban aliados con entidades públicas para la ejecución de la iniciativa.

\section{Análisis del PEIS: el tiempo social a través de sus hitos}

Desde el enfoque de la historia del presente, en la versión elaborada por Aróstegui ${ }^{19}$, una experiencia está constituida por tres momentos: uno es la experimentación de una vivencia, el segundo es la memoria y el tercero es la conciencia. En el último se elabora un proceso particular de interpretación de la vivencia y se construye su significación, que es un proceso de historización de la experiencia vivida.

Entre las fuentes que permitieron construir la historia está la memoria, tanto escrita como oral, de los que participaron en el proyecto. Por lo que la investigación se apoyó tanto de los registros escritos del concurso como en videos y entrevistas relacionados con algunos de los actores

${ }_{19}$ Aróstegui, op. cit., p157 
involucrados y que estaban disponibles en la web. Esas fuentes permitieron analizar el tiempo social del PEIS.

De acuerdo con Gurevitch, el tiempo social, es una categoría que ayuda a comprender las diferencias entre los sujetos o grupos que interactúan dentro de un sistema socio-cultural, es un constructo cultural que constituye parte de la experiencia vivida. Este autor afirma que en toda sociedad existe "no un tiempo cualquiera, único y monolítico, sino toda una gama de ritmos sociales condicionado por las leyes de los diferentes procesos y por la naturaleza de los diversos grupos humanos"20. También plantea que el tiempo social nos permite identificar el actor dominante, que se reconoce por ser quien tiene el control real de la vida social, con una fuerza ideológica influyente.

Para establecer la dominancia del tempo social del PEIS, se partió por identificar los hitos durante la vigencia de ese proyecto y se analizó el actor que impuso el ritmo en cada caso. A continuación, se presentan los cuatro hitos del PIS: la gestación, la primera premiación y el cierre.

\subsection{La gestación de la alianza entre FK y CEPAL}

La alianza entre la CEPAL y la FK se gestó en el marco de los preparativos para la celebración del os 75 de la FK. Fue una iniciativa del Director para América Latina de la FK, Francisco Tancredi, que pretendía conmemorar los 75 años de la FK y hacer un homenaje a su fundador, por lo que dicha entidad se propuso hacer donaciones que tuvieran un significado y permanencia en la región. En una entrevista en 2003, Tancredi afirmó que buscaron un socio con ciertas características: "nos focalizamos en una organización internacional, presente y que tuviera mucha credibilidad en América Latina. Y ahí surgen las organizaciones ligadas a las Naciones Unidas... Llegamos a la conclusión que la CEPAL seria la organización que a nosotros nos parecía la más idónea, para operativizar el concurso" ${ }^{21}$.

Con esa decisión tomada, en 2002 Tancredi se contactó con el Secretario Ejecutivo de la CEPAL de la época, José Antonio Ocampo y le propuso que ejecutara el concurso de innovación social a nivel de américa Latina con el apoyo financiero de la FK. Ambos directivos acordaron los principios generales para el diseño y ejecución del proyecto $^{22}$ y Ocampo decidió alojar el proyecto dentro de su propio cargo, dado lo novedoso de la iniciativa dentro de la CEPAL, pues la tradición de éste organismo público hasta esa fecha era recibir donaciones oficiales de países y no de entidades privadas para financiar proyectos específicos. Además, la CEPAL nunca había realizado un concurso y tenía restricciones legales para entregar premios en dinero.

A partir del 2003, Ocampo nombró una responsable del Proyecto de Experiencias en Innovación Social (PEIS) dentro de la secretaria ejecutiva de la CEPAL a María Elisa Bernal. Pero este proyecto tuvo que ser dividido en dos partes, pues Naciones Unidas nombró a Ocampo en un cargo directivo en Nueva york, por lo que la CEPAL tuvo un cambio de director.

Entre 2003 y 2004 Bernal diseñó las bases para el concurso y formuló el proyecto para llevarlo a cabo con un horizonte temporal de cuatro años, que era el plazo máximo establecido por la FK para sus donaciones. Para esta labor, Bernal se apoyó en tres expertos de la región, a los que invitó a ser parte del Comité Asesor del PEIS. En sus inicios ese Comité estuvo integrado por: Rebeca Grynspan, economista costarricense que ocupaba el cargo de directora de la sede de la CEPAL en México; Nohra Rey de Marulanda, economista colombiana funcionaria del BID; y Eduardo Amadeo, economista argentino, que ocupaba el cargo de embajador de su país en Washington y presidente de la Asociación civil del observatorio social.

\footnotetext{
${ }^{20}$ Gurevitch, op. cit., 274.

21 CEPAL [online], Entrevista a Francisco Tancredi, Santiago, 2007 [consultado en diciembre de 2014], Disponible en internet: <URL:http://www.youtube.com/watch?v=j1esjA68BAE>.

22 Rodríguez y Alvarado, op. cit,37.
} 
Cabe señalar que las tres economistas latinoamericanas ya se conocían desde inicios de los años noventa, cuando Bernal había sido funcionaria del BID (1990- 1994) bajo la jefatura de Rey de Marulanda. Por la misma época que Gryspan habían sido consultoras del BID.

Éste comité precisó los términos del concurso en relación al tipo de organizaciones que podían postular, las áreas temáticas del concurso, los premios a entregar, los lugares y las fechas de premiación, el proceso de evaluación para seleccionar las ganadoras de cada ciclo y la conformación del jurado que elegiría las ganadoras, al que rotularon como el Comité de Notables. Tancredi participó del comité en calidad de observador. Sin embargo, la elaboración del documento que contenía las bases del concurso fue escrito por dos consultores externos: Eduardo Astorga y Nicolás Flaño.

Una vez asumió labores el nuevo Secretario Ejecutivo de CEPAL, José Luis Machinea, se firmó el segundo convenio entre la FK y la CEPAL para ejecutar el concurso. Bajo el mandato de Machinea, el PEIS se instaló en la División de Desarrollo Social (DDS) de la CEPAL, a donde fue trasladada Bernal, quien continuó a cargo del PEIS, con dedicación exclusiva a este proyecto.

En el discurso de la CEPAL, el PEIS se enmarcó dentro de las metas del milenio de las Naciones Unidas y se consideró una fuente de financiamiento alternativo para el desarrollo. Al respecto, la CEPAL en su informe bianual de 2004 resaltó los aportes recibidos de la FK:

"entre 2002 y 2003 las fundaciones, universidades y otras organizaciones del sector privado aumentaron su participación en el total de recursos de la CEPAL por fuentes de financiamiento del 1,2\% al 3,2\%. Los aportes de la FK se registraron como una fuente de recursos extrapresupuestarios, tipo de aporte que la CEPAL caracteriza como potencial, ya que proyecta que probablemente mantenga o aumente su importancia en el futuro" 23 .

De manera que durante la gestación del PEIS, el ritmo del PEIS parecía responder a los ritmos tanto de la FK como de la CEPAL, con apertura a la participación de otros organismos internacionales, como el BID.

\subsection{El lanzamiento del concurso y las ferias de premiación}

Desde su llegada a la CEPAL, José Luis Machinea dio un total respaldo al PEIS. Fue él quien encabezó el lanzamiento del concurso en septiembre de 2004, cuando se realizó un lanzamiento del concurso en la Sede de la CEPAL en Santiago, así como en Buenos Aires, Bogotá, Ciudad de México y Sao Paulo.

En diciembre de 2005 se realizó la primera feria de la innovación social que culminó con la ceremonia de premiación del PEIS, donde el director de la CEPAL y el Presidente de la Junta Directiva del a FK entregaron los premios a las cinco experiencias más innovadoras. Ese año la FK celebró sus 75 años. En ese evento el Secretario Ejecutivo 24 afirmó que los ganadores del concurso "son proyectos que contribuyen, de una u otra manera, al avance de los Objetivos de Desarrollo del Milenio".

En 2006 se realizó al segunda Feria en la Universidad Metropolitana de la ciudad de México con una ceremonia de premiación similar y en 2007 se realizó la tercera feria en la Plaza de las Aduanas en Porto Alegre (Brasil), que contó con la participación del expresidente del BID, Enrique Iglesias. En ambas ocasiones Machinea encabezó la ceremonia de premiación. Ya para la feria del 2008 Machinea había salido de la CEPAL.

23 CEPAL, Actividades de la CEPAL durante el bienio 2002-2003 para promover y apoyar la cooperación técnica entre países y regiones en desarrollo, Santiago, Naciones Unidas, 2004.

24 CEPAL, Palabras del Sr, José Luis Machinea, Secretario ejecutivo de la CEPAL, en ceremonia de premiación del ciclo 2004 2005 [online], Concurso de innovación social, Santiago, 2005 [consultado en diciembre de 2014], Disponible en internet: <URL:http://www.eclac.cl/dds/innovacionsocial/e/concurso.ttm>. 
Dado que según el segundo convenio firmado el PEIS debería durar 4 años, se programó el cierre del concurso para 2008 con la entrega de un trabajo de la sistematización de las lecciones extraídas de las experiencias ganadoras y algunas finalistas hasta el 200725. Ese libro fue elaborado por dos consultores externos contratados para ese fin, en el que se resaltó las 15 experiencias premiadas hasta esa fecha y que aquí se registran en la tabla 2, clasificándolas según categoría y especificando el tipo de actor y lugar.

Tabla 2: Experiencias premiadas entre 205 y 2007 tipo de actor y lugar

\begin{tabular}{|c|c|c|c|}
\hline Categoría & Experiencia premiada & Tipo & Lugar \\
\hline \multirow{5}{*}{$\begin{array}{l}\text { Generación } \\
\text { de ingresos }\end{array}$} & Reforestación Económica Unida y Adensada & $\begin{array}{l}\text { Autogestión } \\
\text { Asociación }\end{array}$ & Rural \\
\hline & Programa integrado de cultivos Andinos & $\begin{array}{l}\text { Autogestión } \\
\text { Cooperativa }\end{array}$ & $\begin{array}{l}\text { Rural- } \\
\text { indígena }\end{array}$ \\
\hline & Let Agogo /leche en abundancia & ONG & Rural \\
\hline & $\begin{array}{l}\text { Manejo y tratamiento de aguas residuales con lenteja } \\
\text { acuática, lechugín y totora }\end{array}$ & $\begin{array}{l}\text { Entidad } \\
\text { pública }\end{array}$ & Rural \\
\hline & $\begin{array}{l}\text { Producción sostenible de truchas en el sistema extensivo } \\
\text { e intensivo de lagunas y jaulas }\end{array}$ & $\begin{array}{l}\text { Autogestión- } \\
\text { Asociación }\end{array}$ & $\begin{array}{l}\text { Rural- } \\
\text { indígena } \\
\end{array}$ \\
\hline \multirow{4}{*}{$\begin{array}{l}\text { Juventud en } \\
\text { riesgo }\end{array}$} & Sistema de sostén para adolescentes tutelados & $\begin{array}{l}\text { Entidad } \\
\text { pública }\end{array}$ & Urbano \\
\hline & $\begin{array}{l}\text { Programa de prevención de violencia a través de } \\
\text { proyectos de resolución de conflictos mediante pares en } \\
\text { escuelas con niños y jóvenes en riesgo y en grupos de } \\
\text { educación no formal }\end{array}$ & $\begin{array}{l}\text { Fundación } \\
\text { privada } \\
\text { comunidad }\end{array}$ & Urbano \\
\hline & Programa para la gestión del conflicto escolar, Hermes & Privado & Urbano \\
\hline & $\begin{array}{l}\text { Prevención del fenómeno de droga y mara en áreas } \\
\text { urbano marginales y rurales }\end{array}$ & $\begin{array}{l}\text { Autogestión } \\
\text { comunidad }\end{array}$ & Urbano \\
\hline \multirow{6}{*}{$\begin{array}{l}\text { Afirmación } \\
\text { de derechos }\end{array}$} & $\begin{array}{l}\text { Trébol de cuatro hojas: estrategia de reducción de la } \\
\text { morbilidad materna, perinatal e infantil }\end{array}$ & Público & Urbano \\
\hline & $\begin{array}{l}\text { Acciones de salud comunitaria en la Floresta Nacional } \\
\text { de Tapajós }\end{array}$ & $\begin{array}{l}\text { Entidad } \\
\text { privada }\end{array}$ & Urbano \\
\hline & Hospedaje estudiantil en familia & $\begin{array}{l}\text { Privado- } \\
\text { publico }\end{array}$ & Urbano \\
\hline & $\begin{array}{l}\text { Programa comunitario de salud- Salud responsabilidad } \\
\text { de todos }\end{array}$ & $\begin{array}{l}\text { Público- } \\
\text { privado }\end{array}$ & rural \\
\hline & $\begin{array}{l}\text { Defensorías comunitarias: una respuesta comunitaria a la } \\
\text { violencia familiar }\end{array}$ & ONG & Urbano \\
\hline & $\begin{array}{l}\text { Programa de erradicación del trabajo infantil y } \\
\text { protección de los adolescentes en el trabajo doméstico }\end{array}$ & ONG & Urbana \\
\hline
\end{tabular}

Fuente: elaboración propia con base en CEPAL, Banco de experiencias en innovación social, 2010.

De esa primera sistematización se resalta que, en la categoría de generación de ingresos, las experiencias ganadoras se localizaban en zonas rurales y el tipo de actor que primaba eran organizaciones autogestionadas de pequeños productores. Además, se resalta que en esa categoría se

${ }^{25}$ La sistematización fue presentada en forma de libro, cuyos autores fueron Adolfo Rodríguez y Hernán Alvarado, con el título Claves de la innovación social en América Latina y el Caribe. 
ubicaron las experiencias que tenían la trayectoria temporal más larga, algunas originadas desde la década de los años ochenta.

En la categoría de juventud en riesgo, las experiencias ganadoras se localizaban en zonas urbanas. Además, el tipo de actor incluía tanto entidades públicas como privadas y alianzas entidades con la comunidad, pero no había presencia de ONGs.

En la categoría de afirmación de derechos los temas eran salud, educación, trabajo y violencia familiar. Entre las experiencias premiadas en los temas de salud y educación, el tipo de actor dominante fueron las alianzas público privadas, mientras que en trabajo y violencia familiar tuvieron presencia las ONGs.

En 2008 se firmó una extensión del convenio entre la FK y CEPAL hasta el 2010, en donde se acordó realizar una ronda más del concurso en 2009 y realizar una clausura del proyecto en 2010. Esta ampliación del PEIS fue firmada por la nueva Secretaria Ejecutiva de CEPAL, Alicia Bárcena, quien fue nombrada en reemplazo de Machinea. Bárcena ya conocía el proyecto pues ella estuvo en el cargo de directora interina cuando se fue Ocampo de la CEPAL y el PEIS apenas estaba en su primera etapa. Ahora volvía cuando el proyecto estaba finalizando.

Por otro lado, el director para la región de la FK se jubiló en 2007 pero no se desvinculo del PEIS sino que paso a ser miembro del Comité de Notables, encargado de elegir a las experiencias ganadoras a partir del 2008.

En 2009 se celebró la última ronda del concurso en la Universidad de san Carlos, en la ciudad de Guatemala y en noviembre 2010 celebraron el cierre del Concurso de Experiencias en Innovación Social en América Latina y el Caribe, en el Instituto de las Américas y en el Instituto Latinoamericano de la Universidad de California, en San Diego, Estados Unidos.

\subsection{La clausura del PEIS}

En 2010 se realizó la ceremonia de cierre del concurso para 2010 en el Instituto de las Américas, en San Diego, Estados Unidos. El lugar de clausura resulta significativo y más si se toma en cuenta que se enmarcó en la celebración de los 80 años de la FK, pues en San Diego se localiza una de las universidades fundadas por FK y la casa de invierno de la familia Kellogg.

Por otro lado, el Instituto de las Américas tiene un valor simbólico pues es un think tank en políticas públicas adscrito a la Universidad de San Diego, que se dedica a promover el dialogo entre líderes industriales y gubernamentales, así como de la academia y la sociedad civil, para fomentar políticas públicas en las Américas. Se destaca el hecho que, en este lugar, la CEPAL y la FK hayan acordado realizar la última feria de experiencias latinoamericanas en innovación social.

Cabe resaltar que a California viajaron los representantes de las 25 experiencias premiadas, así como el equipo de CEPAL encargado del proyecto. Sin embargo, llama la atención la no asistencia de la Secretaria Ejecutiva de CEPAL a la ceremonia de cierre. La persona que pronunció la conferencia magistral de ese evento fue José Antonio Ocampo, que para esa época ya no era funcionario de Naciones Unidos, sino profesor de la Universidad de Columbia.

El acontecimiento que cerró el PEIS en el Instituto de las Américas, fue el lanzamiento de un documento que sintetizaba los resultados del proyecto con énfasis en lecciones de política pública, extraídas de las 25 experiencias premiadas (las que se registran arriba en la tabla 1). Esta nueva publicación fue titulada De la innovación social a la política pública, y tuvo como subtítulo Historias de éxito en América latina y el Caribere.

26 Nohra Rey de Marulanda y Francisco Tancredi, De la innovación social a la política pública, Documento de proyecto, Santiago, CEPAL, Naciones Unidas, 2010. 
En esta segunda publicación, la clasificación de las experiencias ganadoras registró un ligero cambio ligeramente respecto al registro del libro escrito por Rodríguez y Alvarado. La categoría de afirmación de derechos se subdividió en dos: por un lado, salud y educación, y por otro, voluntariado, responsabilidad social y participación comunitaria. Al comparar los datos de las Tablas 1 y 2 , es de notar que sólo una experiencia se reclasificó (la de defensorías comunitarias): pasó de haber sido clasificada en afirmación de derechos al grupo de voluntariado.

\subsection{Los vínculos del PEIS dentro de la CEPAL}

Cuando se revisan los archivos de la CEPAL en el periodo de vigencia del proyecto, se constata que El PEIS figuraba como un proyecto autónomo, sin mayores vínculos con otros, a acepción de un trabajo sobre innovación social y desarrollo local realizado con apoyo del ILPES ${ }^{27}$. Además, el PEIS se caracterizó por la baja participación de los funcionarios de la entidad, por lo menos en cuanto a sus publicaciones.

Los dos libros que fueron publicados como parte del PEIS estuvieron a cargo de personas externas a la CEPAL. El primer libro fue Claves de la innovación social, publicado en 2008, que fue escrito por dos consultores externos contratados, Rodríguez y Alvarado. La segunda publicación fue De la innovación social a la política pública. Historias de éxito en América Latina y el Caribe, que fue escrito por dos miembros del comité de notables, Nohra Rey de Marulanda y Francisco Tancredi. Para la fecha en que salió la publicación Rey ya era exfuncionaria del BID y Tancredi era exfuncionario de la FK. Esta segunda publicación no fue considerada un libro de CEPAL, sino que quedó registrado como documento de proyecto, que no tuvo revisión editorial ni contó con la firma de la Secretaria Ejecutiva.

Ambas publicaciones retomaron los aportes de las experiencias ganadoras y contribuyen a los objetivos del PEIS, en la medida que entregan un reconocimiento al aporte de las experiencias y realizan difusión de sus logros. Pero esas las publicaciones se quedan limitadas al proyecto, sin llegar a configurar un aporte conceptual o de proceso. No discuten el concepto de innovación social ni entran en el debate en torno al mismo, que ya para el 2010 contaba con amplia literatura a nivel mundial. Tampoco entregan una explicación de lo que entiende por proceso social, sino que reproduce la ambigüedad del término. Hernández, Tirado y Ariza señalan que "no existe una definición que goce de consenso ni siquiera entre quienes idean y ponen en práctica las innovaciones sociales, pues no es posible discernir en muchos casos si se trata de productos, políticas públicas, formas organizativas de la sociedad civil o todas ellas y, otras posibles, a la vez" 28 .

Por otro lado, aunque el libro de Rodríguez y Alvarado hace un esfuerzo por sistematizar los aprendizajes alcanzados por los actores locales a partir de la opinión de ellos mismos y derivar enseñanzas para los formuladores de políticas públicas, queda incompleto o malogrado porque no explica cómo emergen las necesidades sociales que dieron origen a las innovaciones sociales. Esta carencia del PEIS es aún más notoria dada la trayectoria de la CEPAL como centro de pensamiento en la región que tiene un legado de pensamiento histórico estructuralista. Era esperable que la CEPAL produjera alguna publicación vinculando al PEIS con el origen de las experiencias innovadoras o con los procesos históricos y las transformaciones vividas en la región. Más si se toma en cuenta que varias de las experiencias ganadoras del PEIS tuvieron origen en las décadas de los ochenta y noventa, que fue un periodo de fuertes transformaciones económicas, políticas y sociales en la región.

\footnotetext{
${ }^{27}$ Luz Angela Rodríguez, María Elisa Bernal y Mauricio Cuervo, "Innovación social y desarrollo económico local”, CEPAL Serie de politicas sociales 170, Santiago, 2011.

28 José Hernández, Pilar Tirado y Antonio Ariza, "El concepto de innovación social: ámbitos, definiciones y alcances teóricos", CIRIEC-España, No. 88, 2016, 165-199.
} 
Además, se resalta que la CEPAL como centro de investigación, cuenta con personal calificado con reconocidas trayectorias de los que se podría esperar un análisis alternativo de las experiencias que participaron del PEIS, por ejemplo, el caso del mismo director de la División de Desarrollo Social, Martín Hopenhayn.

De manera que la ausencia de publicaciones de funcionarios de CEPAL relativas la PEIS y el hecho que los dos libros del proyecto fueran escritos por personas externas a la entidad, son indicios del carácter aislado que tuvo en proyecto dentro de esta entidad. En cambio, para la FK, el PEIS aparece como un producto atractivo, que mereció la ampliación del plazo máximo que ellos otorgaban a sus donaciones.

\section{El dominio del tiempo social}

En primer lugar, se destaca a quien mayor peso tuvo a la hora de establecer los hitos del proyecto en función de los propios fue la FK. Las ceremonias acontecieron en las fechas y lugares que convenían a sus actividades: la primera premiación se programó para que aconteciera en el año de su aniversario 75, y el cierre del proyecto ocurrió en su aniversario 80, que además se realizó en la ciudad donde la FK tiene una presencia significativa.

Además, es significativo que un concurso de experiencias latinoamericanas no celebrase su cierre dentro de la región, sino en el país de origen de la FK. Así mismo, resulta significativo que la clausura del proyecto tuviera lugar en un centro de pensamiento distinto a la CEPAL, pues fue el momento de entrega de recomendaciones de políticas para la región y con la elección de ese lugar, se alimentaba la creencia que las políticas vienen del norte.

La revisión de los hitos del PEIS permite detectar detalles poco visibles, si el proyecto se revisa sólo a partir de las publicaciones, pues en ellas se expresa la idea que el proyecto fue liderado por la CEPAL y que la FK actuó solo como ente financiador.

En segundo lugar, se destaca que el dominio ejercido por la FK no tuvo resistencia por parte de CEPAL, sino que desde le inició se pactó las condiciones para que el PEIS operase como un proyecto autónomo. Pero más que autónomo, podríamos decir que operó como un enclave dentro de la CEPAL, ya que no generó lazos con otros proyectos o equipos de investigación dentro de esa entidad y cuando cerró no hubo ninguna unidad o persona que acogiera los aportes del PEIS o que diera alguna continuidad al trabajo realizado. En ese sentido, la CEPAL ejerció un rol de gestor de proyectos más que de centro de pensamiento, pues no construyo un vínculo entre los resultados del PEIS y los planteamientos sobre desarrollo en la región.

En tercer lugar, es de notar que la CEPAL adoptó un concepto práctico de innovación social que fue coherente con la mecánica del PEIS, pero no profundizó en él ni en los procesos sociales de las experiencias. No vinculo las experiencias locales con los procesos históricos estructurales de la región. De manera que la CEPAL desaprovecho su potencial institucional y el esfuerzo de sistematización de experiencias locales. Los que sacaron alguna ventaja de ese descuido fueron los consultores externos, que operaron en la lógica del ente financiador.

En cuarto lugar, los recurrentes cambios de directivas en CEPAL en el periodo 2002-2010 y las distintas posturas de los directores respecto la PEIS, dejan en evidencia ciertos comportamientos erráticos en materia de orientación de las políticas públicas, que pude ser indicio que el desarrollo social era un campo de experimentación dentro de la entidad, en el que se gestionaban diversos proyectos y se ensayaban alternativas pero donde la entidad no se hacía cargo de asegurar una correspondencia con una estrategia de desarrollo económico para la región.

Finalmente, el análisis realizado invita a una reflexión sobre la imagen que la última publicación del PEIS instaló de las experiencias como "historias de éxito", tal como se evidencia en el subtítulo del 
documento. Pues ese fue un registro que realizaron expertos de la región que no están adscritos a la CEPAL sino al BID y a la FK. Aunque en la introducción del documento declaran buscar el rescate del aporte de las organizaciones locales, ellos subordinan la vOz de sus protagonistas y descuidan las narrativas propias de las organizaciones locales, otorgando primacía al discurso tecnocrático propio de los organismos financieros internacionales.

\section{Conclusiones}

Desde el enfoque de la historia del presente, el análisis del tiempo social del PEIS permite concluir que quien dominó el ritmo del proyecto fue la FK, que fue quien gestó la idea, impuso el ritmo del tiempo social, aunque sin definirlo, marcó las fechas en las que se produjeron los hitos del PEIS y participó de la formulación y registro de lecciones de política que entregó el proyecto.

Por otro lado, la CEPAL asumió un rol más de administrador que de líder del proyecto. El PEIS fue una especie de enclave dentro de la División de Desarrollo Social de la CEPAL que tuvo poca articulación con otras iniciativas en ese espacio y se fue quedando al margen del debate en torno al desarrollo social de la región, aislación que fue más notoria con el cambio de dirección ejecutiva.

La CEPAL de manera más bien tácita avaló el registro de las experiencias como "historias de éxito" en materia de innovación social, sin entregar un marco de reflexión para comprender cómo ellas se relacionaron con las transformaciones económicas, sociales y políticas ocurridas en la región.

En el contexto regional de inicios del siglo XXI y ante las tendencias de los organismos internacionales referentes a abrieron espacios para la participación de los actores privados en el campo del desarrollo social, el PEIS aparece como el resultado del esfuerzo de un actor privado por legitimar su imagen en la región y facilitar su participación en inversiones sociales y no como una iniciativa del ente público por encontrar nuevas políticas públicas que permitieran enfrentar los desafíos de desarrollo social.

El PEIS fue un caso de alianza público privado transnacional dominado por la FK, que también hubiera podido contribuir a los objetivos del ente público, si la CEPAL hubiese optado por ejercer el liderazgo del proceso, cosa que no ocurrió.

Finalmente, la investigación invita a reflexionar sobre las consecuencias de las alianzas públicoprivadas dentro de una lógica de proyectos, pues como se mostró en el caso del PEIS, puede conducir a una subordinación de lo público a lo privado a nivel transnacional, donde el ente financiador ejerce dominio y el ente público pierde autonomía y autenticidad.

\section{Bibliografía}

\section{Fuentes impresas}

ARÓSTEGI, Julio. La historia vivida: sobre la historia del presente. Madrid: Alianza, 2004.

BANCO MUNDIAL, Informe sobre el desarrollo mundial 2000/2001: lucha contra la pobreza, Madrid, MundiPrensa, 2001.

BÉRTOLA, Luis y OCAMPO, José Antonio. El desarrollo de América Latina desde la independencia, México, Fondo de Cultura Económica, 2013.

BIELSCHOWSKY, Ricardo. Sesenta años de la CEPAL: estructuralismo y neoestructuralismo, Revista de la CEPAL, 1998, 173-194.

BONI, Alejandra. "El sistema de la cooperación internacional al desarrollo. Evolución histórica y retos actuales", Carola Calabuig Torno y María de los Llanos Gómez-Torres (coords), La 
cooperación internacional para el desarrollo, Cuadernos de cooperación para el desarrollo No 1, Valencia, Univeristat politécnica de Valencia, 2010, 7-52.

CEPAL, Actividades de la CEPAL durante el bienio 2002-2003 para promover y apoyar la cooperación entre países y regiones en desarrollo. Santiago, Naciones Unidas, 2004.

CEPAL, Panorama social, Santiago, Naciones Unidas, 2010.

CEPAL, Panorama social. Santiago, Naciones Unidas, 2009.

FISHER, Karin y PLEHWE, Dieter. Redes de think tank e intelectuales de derecha en América Latina, Revista Nueva Sociedad, 215, 70-86.

FUNDACION W.K. KELLOGG. A legacy of innovation, Michigan, W.K. Kellogg, 2004.

GUREVITCH, Aron. "El tiempo como un problema de historia cultural”. En Paul Ricoeur, Claude Larre, Raimundo Panikkar, Alexis Kagame, G.E.R Lloyd, André Neher, Germano Pattaro, Louis Gardet y Aron Guervitch (eds), Las culturas y el tiempo, Salamanca, UNESCO, 1979, 260281.

HERNÁNDEZ, José, TIRADO, Pilar y ARIZA, Antonio. "El concepto de innovación social: ámbitos, definiciones y alcances teóricos, CIRIEC, España, No. 88, 2016, 165-199.

McGANN, Joseph, Global go to think tank index report, Pennsylvania, University, 2016.

MARTIN, Maximilian. Cuatro revoluciones en la filantropia global, Working papers, Ginebra, Impact Economy, 2011.

OCAMPO, José Antonio. La crisis latinoamericana de la luz de la historia. En José Antonio Ocampo, Bárbara. Stallings, Inés. Bustillo, Helvia. Belloso, y Roberto. Frenkel, La crisis latinoamericana de la deuda desde la perspectiva histórica, Santiago, Naciones Unidas, 2014.

PRAHALAD, C.K, y HARD, Stuart. "The fortune at the bottom of the pyramid", Security and strategy, 2002.

PREBISCH, Raúl. Exposición de Raúl Prebisch. Notas y Cometarios, Dos exposiciones en La Paz: Raúl Prebisch y Gabriel Valdés, Revista de la CEPAL, 9, 1979:75-77.

REY DE MARULANDA, Nohra y TANCREDI. Francisco, De la innovación social a la politica pública, Santiago, Naciones Unidas, 2010.

RODRÍGUEZ, Adolfo y AVARADO, Hernán, Claves de la innovación social en América latina y el Caribe, Santiago, Naciones Unidas, 2008.

RODRÍGUEZ Luz Angela, BERNAL, María Elisa y CUERVO, Mauricio, "Innovación social y desarrollo económico local", CEPAL Serie de politicas sociales 170, Santiago, 2011.

STALLING, Bárbara. "La economía política de las negociaciones de la deuda: América Latina en la década de los ochenta”, José Antonio Ocampo, Bárbara Stallings, Inés Bustillo, Helvia. Belloso, y Roberto. Frenkel, La crisis latinoamericana de la deuda desde la perspectiva histórica, Santiago, Naciones Unidas. 2014.

WILLIAMSON, John, "Lo que Washington quiere decir cuando se refiere a reformas de políticas económicas", Manuel Guitián y, Joaquim Muns (eds), La cultura de la estabilidad y el consenso de Washington, Colección de estudios e informes, No 15. Barcelona, La Caixa, 1999.

\section{Fuentes electrónicas y digitales}

CEPAL, Banco de experiencias en innovación social [online], Experiencias en innovación social, Santiago, 2010 [consultado en noviembre de 2018], Disponible en internet: <URL:

https://dds.cepal.org/innovacionsocial/e/experiencias.htm>

CEPAL, Palabras del Sr, José Luis Machinea, Secretario ejecutivo de la CEPAL, en ceremonia de premiación del ciclo 2005 -2006 [online], Concurso de innovación social, Santiago, 2004 [consultado en diciembre de 2014], Disponible en internet: <URL:http://www.eclac.cl/dds/innovacionsocial/e/concurso.ttm>. 
Luz Rodríguez: El tiempo social de las alianzas público privadas transnacionales en América Latina: un estudio de caso (2002-2010), Izquierdas, 49, enero 2020:104-122

CEPAL. Palabras del Sr, José Luis Machinea, Secretario ejecutivo de la CEPAL, en ceremonia de premiación del ciclo 2004 -2005 [online], Concurso de innovación social, Santiago, 2005 [consultado en diciembre de 2014], Disponible en internet: <URL:http://www.eclac.cl/dds/innovacionsocial/e/concurso.ttm>.

CEPAL. Palabras del Sr, José Luis Machinea, Secretario ejecutivo de la CEPAL, en ceremonia de lanzamiento del concurso [online], Concurso de innovación social, Santiago, 2004 [consultado en diciembre de 2014], Disponible en internet: <URL:http://www.eclac.cl/dds/innovacionsocial/e/concurso.ttm>.

CEPAL. Entrevista a Francisco Tancredi, [online], Santiago, 2007 [consultado en enero de 2014], Disponible en internet: <URL:http://www.youtube.com/watch?v=j1esjA68BAE>. 